\title{
Heart rate response profiles during head upright tilt test in infants with apparent life threatening events
}

\author{
A Edner, M Katz-Salamon, H Lagercrantz, J Milerad
}

\begin{abstract}
Sympatheticovagal imbalance causing episodes of severe bradycardia has been suggested as a cause of apparent life threatening events (ALTEs). The autonomic control of the heart rate in 18 infants with ALTEs and 12 controls was evaluated by the head upright tilt test. Five different heart rate response profiles (compared with the baseline) were observed during the tilt: (1) increase followed by a decrease and return to baseline; (2) sustained increase; (3) decrease followed by an increase and return to baseline; (4) sustained decrease; (5) no change. Eighty eight per cent of controls responded with heart rate increase followed by decrease or sustained increase compared with $55 \%$ of infants with an ALTE; a significantly greater proportion of infants with ALTEs than controls responded with heart rate decrease or no change in rate $(45 \% v 8 \%)$. This altered reaction during a head upright tilt test may be an expression of an underlying autonomic dysfunction in infants who have experienced an ALTE.

(Arch Dis Child 1997;76:27-30)
\end{abstract}

Keywords: sudden infant death syndrome; apparent life threatening events; heart rate.

Infants who experience apparent life threatening events (ALTEs) are generally regarded to be a group with an increased risk of dying of sudden infant death syndrome (SIDS)..$^{12}$ Although the precise link between SIDS and ALTEs has not been conclusively established, most investigators favour the view that an ALTE may be an abortive form of SIDS, ${ }^{3}$ and physiological studies of infants with ALTEs may thus provide insight into the pathophysiology of SIDS. Irregular breathing, prolonged apnoea, and repeated bradycardia during sleep are more common in infants experiencing an ALTE than in age matched controls. ${ }^{46}$ This instability in cardiorespiratory function appears to be related to a general autonomic imbalance. Increased vagal tone, as evidenced by an exaggerated bradycardia in response to eyeball pressure ${ }^{7}$ or laryngeal water instillation ${ }^{8}$ and excessive night sweats ${ }^{9}$ suggestive of increased sympathetic tone, have both been demonstrated in infants with ALTEs.

A practical problem when investigating infants after an ALTE is that most tests used to assess sympatheticovagal balance are not well suited for clinical practice. Laryngeal water instillation is technically difficult and potentially hazardous, and the oculocardiac reflex may be painful and difficult to perform in a standardised fashion.

The head upright tilt table test has gained increasing acceptance as a tool for examination of children with neurocardiogenic syncope of unknown origin. ${ }^{10}$ In infants the test is performed as a tilt cot test, that is, as a quick positional change while lying in a cot.

The purpose of the present study was to compare the heart rate response to head upright tilt in infants who had suffered an ALTE with those of age matched controls.

In contrast with a previous study where the response was evaluated in quantitative termsthat is, as the percentage change in heart rate compared with resting levels ${ }^{11}$ - we chose to characterise the dynamics of the response in qualitative terms as a heart rate response profile.

Eighteen infants, consecutively referred for investigation after an ALTE, were compared with age matched babies prospectively recruited from a normal nursery.

\section{Subjects and methods}

INFANTS

Index cases

Eighteen infants, mean (SD) age 8.7 (6.4) weeks, who had experienced one or several ALTEs, were admitted to the study. An ALTE was defined as a sleep related apnoeic episode combined with pallor or cyanosis and loss of muscle tone, which required vigorous stimulation or mouth to mouth resuscitation.

Before the investigation, other possible causes of the event such as infection or neurological, cardiac, or metabolic disorders were ruled out during a three day observation period. The infants were then referred to our unit for the tilt table test, which was usually performed within a week of the ALTE.

\section{Controls}

The control group consisted of 18 infants prospectively recruited in a normal nursery. Parents of newborn babies who matched an ALTE case for birth order, sex, and gestational age were asked to participate when their baby was at the same age as the ALTE infant. Eighteen parental couples agreed to participate, but only 12 of these finally showed up for the investigation, which was performed at the age 
at which the ALTE had occurred. Therefore the comparison was based on evaluation of differences between groups. All control infants had a normal physiological examination at the first and fifth day of life.

Informed parental consent was obtained in all cases and the protocol was approved by the local ethical committee.

\section{PROTOCOL}

The infants were admitted to the laboratory between 9 and $10 \mathrm{am}$. Room temperature was kept at $22^{\circ} \mathrm{C}$. Electrodes for a six lead electrocardiograph were positioned on the left and right upper arm and the corresponding leg. After feeding, the infants were nursed to sleep supine lightly dressed with the electrodes on. The test started when the baby was lying quietly with eyes closed.

\section{Equipment}

The tilting bed consisted of a plastic bassinet hinged on two metal pegs. The pegs rested on a steel frame, on which the bassinet was locked in place with a latch. When unlatched the bassinet could be quickly tilted from a horizontal position to $45^{\circ}$ head-up. An electrocardiogram (ECG) was recorded with a Siemens Elema Mingograf 62, at a paper speed $50 \mathrm{~mm} / \mathrm{s}$ (Siemens Elema, Stockholm, Sweden).

Tilting procedure

After a five minute baseline recording period during which the infant remained quiet and the ECG free from movement artefacts, the bed was rapidly tilted (in one to two seconds) until a position of $45^{\circ}$ head-up was reached. The ECG was then recorded for an additional minute in the tilted position. Thereafter the cot was returned to the horizontal position.

Data analysis

The changes in heart rate in response to the head upright tilt table test were analysed using the following steps. Consecutive heart beats during the tilt were grouped into five beat intervals and the mean of each five beat interval was calculated. Each five beat interval meanwas compared with a 40 beat reference period before the tilt to ascertain whether the five beat rate was higher, lower, or equal to the reference period (see under statistical methods). A heart rate profile during the test was next created by plotting the deviation from the reference period of each five beat interval. The profile was thus based on whether or not the five beat means were significantly different from the resting mean. In addition the relation between the longest and shortest RR interval, the ratio maximum/minimum heart rate, during the tilt was also calculated.

STATISTICAL METHODS

Beat to beat variability during the reference period and during the tilt were tested for goodness of fit to normal distribution $\left(\chi^{2}\right.$ test) for each infant. The mean value for each five beat interval was compared with the reference heart rate by analysis of variance, the test for homogeneity, which calculates the least significant difference.

The difference between the reference heart rate in control infants and those with an ALTE was tested for significance by analysis of variance.

The ratio maximum/minimum beats per minute (bpm) in infants with an ALTE and controls was tested for significance with the Kolmogorov-Smirnov two sample test.

\section{Results}

The group results are presented as mean (SD). The mean heart rate preceding the tilt was similar in babies with ALTEs and controls (133 (16) and 145 (16) bpm respectively, $p=0.12$ ). The ratio maximum/minimum heart rate during tilt was also similar in the two groups $(\mathrm{p}=0.34)$.

The heart rate response to tilting could be grouped into five distinct profiles (fig 1). (1) Heart rate increased and this was followed by a decrease and then a return to baseline. This response was seen in four $(22 \%)$ infants with an ALTE and 10 (83\%) controls. Mean baseline heart rate was 132 (19) bpm and the maximal increase from the reference was 24 (11) bpm. The following decrease was 13 (11) bpm below the reference heart rate. (2) Sustained heart rate increase was seen in six $(33 \%)$ babies with an ALTE and one $(8 \%)$ control. Mean baseline heart rate in this group was 147 (21) bpm and the increase 26 (14) bpm. (3) Heart rate decreased and this was followed by an increase and then a return to baseline. This pattern was found in three patients with an ALTE and one control. The mean baseline was 150 (5) bpm and the initial decrease of 19 (7) bpm was followed by an increase of 16 (5) bpm. (4) Continuous heart rate decrease was seen in three infants with an ALTE only. The mean baseline of 163 (1) bpm was followed by a decline in heart rate by 25 (6) bpm. (5) No change in heart rate was observed in two of the infants with an ALTE in response to tilt (mean baseline 128 (17) bpm).

\section{Discussion}

The key finding of this study is that infants with ALTEs have a qualitatively altered heart rate reaction to head up tilt when compared with age matched controls. While $83 \%$ of the control babies showed a response of a transient heart rate increase followed by a fall, this pattern was seen in only $22 \%$ of infants with an ALTE (table 1). Constant heart rate decrease or no change were patterns seen in $28 \%$ of babies with ALTEs, but in none of the controls. These findings, suggestive of a lower sympathetic stimulation of heart rate in response to positional changes, are in agreement with a number of previous observations demonstrating an autonomic imbalance in infants with an ALTE. ${ }^{811-13}$

The head upright tilt table test is increasingly used as a diagnostic tool for evaluation of adults and children with neurocardiogenic syncope of unknown origin. ${ }^{14}{ }^{15}$ These patients are believed to be unable to maintain a normal 

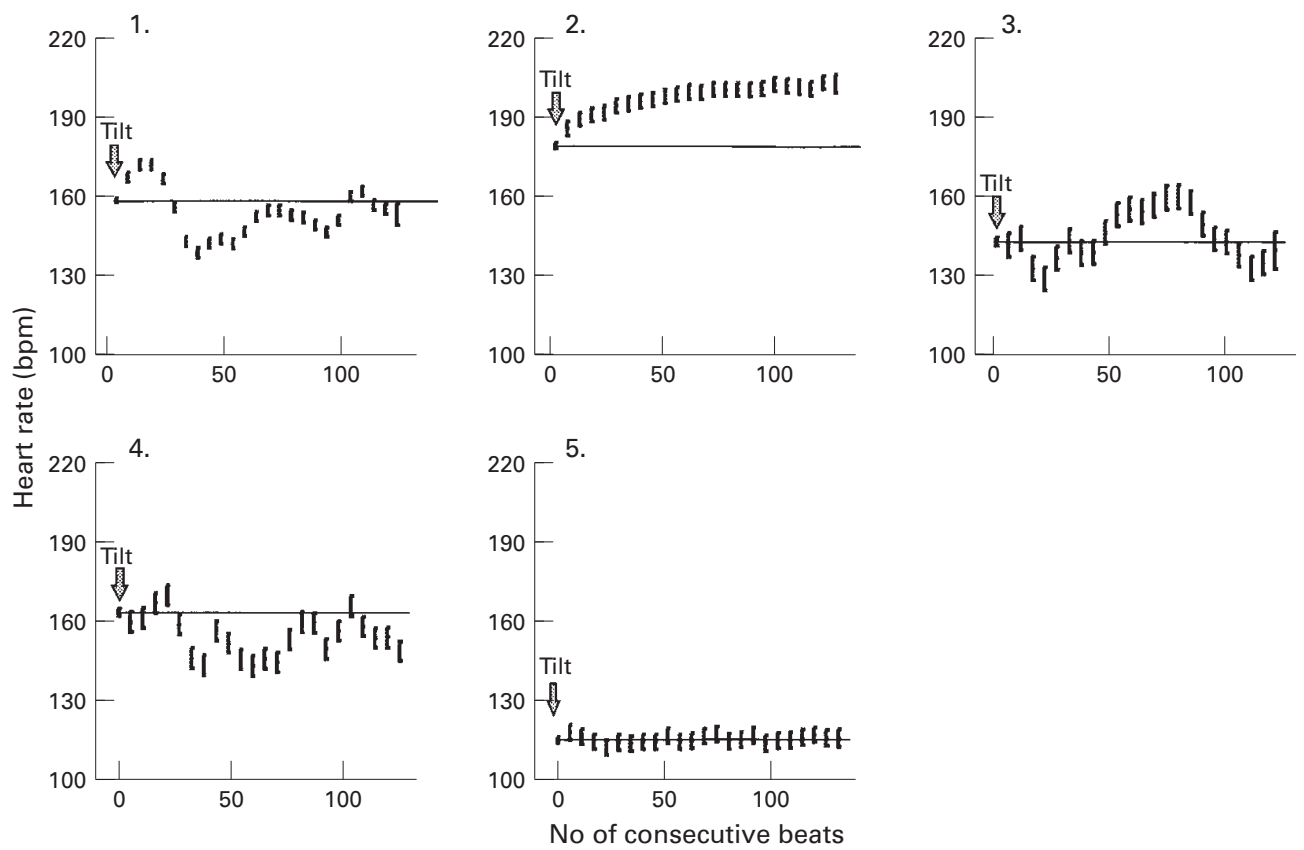

Figure 1 Dynamic heart rate response profiles to head upright tilt table test. The diagrams show instantaneous changes in heart rate during the first 120 heart beats after the tilt. The horizontal line marks the mean heart rate of 40 beats preceding the tilt and the arrow over the first dot indicates where the tilt starts. Each symbol represents a five beat mean (SD). Profile 1: heart rate increase followed by decrease and return to baseline. Profile 2: sustained heart rate increase. Profile 3: heart rate decrease followed by increase and return to baseline. Profile 4: sustained heart rate decrease. Profile 5: no change in heart rate.

cardiac output during the test due to a failure to maintain a balance between the sympathetic and vagal modulation of heart rate and blood pressure in response to changes in body posture. ${ }^{14}$

A change in body position from horizontal to head up results in gravitationally mediated pooling of blood in the lower extremities, a decrease in venous return, and a lower ventricular filling rate. This evokes a decrease in aortic and cardiopulmonary baroreceptor discharge which results in an increase in sympathetic activity and withdrawal of vagal tone. The net effect is a compensatory increase in heart rate and blood pressure. In normal subjects the decrease in baroreceptor discharge associated with head upright tilt is also accompanied by a reduced afferent activity of the mechanoreceptor $\mathrm{C}$ fibres located in the atrial ventricles and pulmonary artery. The decrease in $\mathrm{C}$ fibre discharge promotes the increase in sympathetic tone and vagal deactivation. ${ }^{16}$

Subjects suffering from neurocardiogenic syncope have a more profound decrease in venous return after head upright tilt and respond with an exaggerated increase in ventricular contraction force. The increased

Table 1 Prevalence of heart rate (HR) profiles in infants with an ALTE and controls; results are number (\%)

\begin{tabular}{lll}
\hline HR response to tilting & Infants with an ALTE (n=18) & Controls $(n=12)$ \\
\hline $\begin{array}{l}\text { 1. HR } \uparrow \text { followed by HR } \downarrow \text { and return to } \\
\text { baseline }\end{array}$ & $4(22)$ & $10(83)$ \\
$\begin{array}{l}\text { 2. Sustained HR } \uparrow \\
\text { 3. HR } \downarrow \text { followed by HR } \uparrow \text { and return to }\end{array}$ & $6(33)$ & $1(8)$ \\
$\quad$ baseline & $3(17)$ & $1(8)$ \\
4. Sustained HR $\downarrow$ & $3(17)$ & 0 \\
5. No change in HR & $2(11)$ & 0
\end{tabular}

Profiles 1 and 2 were significantly more frequent in controls than in babies with an ALTE $\left(\chi^{2}=\right.$ $7.11, \mathrm{p}=0.028)$. Profiles 4 and 5 were only seen in infants with an ALTE $(28 \%)$. myocardial tension activates cardiopulmonary mechanoreceptors in a similar way to the reaction to a hypertensive episode. This conflicting feedback information from baroreceptors and mechanoreceptors results in a rapid withdrawal of sympathetic tone and a decrease in Heart rate and hypotension, that is a paradoxical and inappropriate response to the original stimulus.

This scenario, although well documented in adults, ${ }^{16}{ }^{17}$ has not yet been conclusively confirmed in infants under 1 year of age. However, since comparable physiological mechanisms are operational during the first months of life, it seems reasonable to hypothesise that the pathophysiology of ALTEs is similar to neurocardiogenic syncope, that is an inappropriate or sluggish balance between vagal and sympathetic activation.

Several investigators have found that infants who have experienced an ALTE have an exaggerated response to vagal stimulation, as evidenced by a marked bradycardia in response to oculobulbar pressure. ${ }^{18}$ At the same time symptoms more attributable to an increased sympathetic activity such as profuse night sweats have been noted. ${ }^{9}$ Although seemingly contradictory, these results of both vagal and sympathetic hyperactivity are in agreement with the present findings suggesting a sympatheticovagal imbalance rather than vagal or sympathetic hyperactivity alone.

In a previous study utilising the head upright tilt table test, the investigators used the maximum fall in blood pressure and heart rate after the tilt manoeuvre to compare infants with ALTEs to age matched controls. ${ }^{11} \mathrm{Al}-$ though both investigations are in agreement with regard to the observation that infants with ALTEs respond differently from controls, there 
are methodological differences in the evaluation of the response.

The previous study of Fox and Matthews utilised a quantitative approach - that is, the percentage decrease in heart rate and blood pressure after the tilting manoeuvre was used to characterise the response. ${ }^{11}$ The present study suggests that a number of infants with ALTE may have a qualitatively altered response without having a marked fall in heart rate after the tilt - that is, a 'normal' response by quantitative criteria. It therefore appears that a qualitative analysis of the dynamics of the response may offer additional information not readily available when only quantitative changes are taken into account.

In conclusion it was found that most infants who have experienced an ALTE have a qualitatively altered heart rate response to the head upright tilt table test, a finding suggestive of an imbalance between the cardiovascular vagal and sympathetic tone. The head upright tilt table test appears to be an easy and safe bedside test, with a higher specificity than conventional vagal provocation.

This study was supported by the First of May Flower National Campaign for Children's Health, Swedish SIDS association, Samariten Foundation, General Maternity Hospital Foundation and Swedish Medical Research Council grant No 11265.

1 Keens TG, Ward SL. Apnea spells, sudden death, and the role of the apnea monitor. Pediatr Clin North Am 1993;40:897-911.

2 Wennergren G, Milerad J, Westphall I, Tunell R. Consensus statement on clinical management. Acta Paediatr Scand 1993;82(suppl 389):114-6.

3 Kahn A, Groswasser J, Sottiaux M, Rebuffat E, Franco P. Clinical problems in relation to apparent life-theart rateeatening events in infants. Acta Paediatr Scand 1993; 82(suppl 389):107-10

4 Kahn A, Montauk L, Blum D. Diagnostic categories in infants referred for an acute event suggesting near-miss SIDS. Eur $\mathcal{F}$ Pediatr 1987;146:458-60.

5 Kahn A, Blum D, Montauk L. Polysomnographic studies and home monitoring of siblings of SIDS victims and of infants with no family history of sudden infant death. Eur $\mathcal{F}$ Pediatr 1986;145:351-6.

6 Hunt CE, Brouillette RT. Sudden infant death syndrome: 1987 perspective. F Pediatr 1987;110:669-78.

7 Lucet V, Do Ngoc D, Cauchemez B, et al. Treatment of Lucet V, Do Ngoc D, Cauchemez B, et al. Treatment of
reflex vagal hypertonia in infants. Role of diphemanil (apropos of 50 cases). Arch Fr Pediatr 1987;44:359-63.

8 Wennergren G, Hertzberg T, Milerad J, Bjure J, Lagercrantz $\mathrm{H}$. Hypoxia reinforces laryngeal reflex bradycardia in infants. Acta Paediatr Scand 1989;78:11-7.

9 Kahn A, Van de Merckt C, Dramaix M, et al. Transepidermal water loss during sleep in infants at risk for sudden death. Pediatrics 1987;80:245-50.

10 Samoil D, Grubb BP, Kip K, Kosinski DJ. Head-upright tilt table testing in children with unexplained syncope. Pediatrics 1993;92:426-30.

11 Fox GP, Matthews TG. Autonomic dysfunction at different ambient temperatures in infants at risk of sudden infant death syndrome. Lancet 1989;ii: 1065-7.

12 Matthews TG. The autonomic nervous system-a role in sudden infant death syndrome. Arch Dis Child 1992;67: 654-6.

13 Kahn A, Rebuffat E, Sottiaux M, Muller MF. Recent advances in sudden infant death syndrome: possible autonomic dysfunction of the airways in infants at risk. Lung 1990;168(suppl):920-4IS

14 Kosinski DJ, Grubb BP. Neurally mediated syncope with an update on indications and usefulness of head-upright tilt table testing and pharmacologic therapy. Curr Opin Cardiol 1994;9:53-64.

15 DiMario FJ Jr, Burleson JA. Autonomic nervous system function in severe breath-holding spells. Pediatr Neurol 1993;9:268-74.

16 Kosinski D, Grubb BP, Temesy-Armos P. Pathophysiological aspects of neurocardiogenic syncope: current concepts and new perspectives. PACE Pacing Clin Electrophysiol 1995;18:716-24.

17 Grubb BP, Gerard G, Roush K, et al. Cerebral vasoconstriction during head-upright tilt-induced vasovagal syncope. A paradoxic and unexpected response. Circulation 1991;84: 1157-64.

18 Kahn A, Riazi J, Blum D. Oculocardiac reflex in near miss for sudden infant death syndrome infants. Pediatrics 1983; 71:49-52. 